\title{
Präferenzen, Interesse(n) und Gründe von SchülerInnen für das Erlernen von (Fremd-)Sprachen Eine Pilotstudie an Kärntner Schulen
}

\author{
Magdalena KaLtSEIS \\ University of Klagenfurt (Austria) \\ Ursula Doleschal \\ University of Klagenfurt (Austria)
}

\begin{abstract}
Recently, multilingualism and linguistic diversity have become one of the most important educational concerns in Austria. For many Austrian schoolchildren, the official state language German is not their first language and this fact is a challenge for many schools across the country. In this respect, schools in the federal province of Carinthia are of particular interest as Carinthia lies in the trilingual Alps-Adriatic region. Therefore, the current study investigates language diversity at Carinthian schools by asking schoolchildren which foreign
\end{abstract} languages they would be interested in learning and for what reason. Thereby, this study wants to highlight linguistic plurality in schools and plead for a greater variety of and openness for (foreign) languages taught and spoken at school.

Keywords: language policy, bilingualism, multilingualism, foreign language education, Slavic languages, school, Carinthia

(c) by the authors; magdalena.kaltseis@aau.at; ursula.doleschal@aau.at

Colloquium: New Philologies, Volume 5, Issue 2 (2020)

doi: 10.23963/cnp.2020.5.2.3

Stable URL: https://colloquium.aau.at/index.php/Colloquium/article/view/134

This work is licensed under a Creative Commons Attribution 4.0 International License (CC BY 4.0). 
Schule und Mehrsprachigkeit sind eng miteinander verbunden, denn in diesen „Brennpunkte[n] der Kommunikation“" (Schreger 2013, 15) treffen Schüler und Schülerinnen verschiedenster Herkunft und mit unterschiedlichen sprachlichen Kenntnissen aufeinander. Spätestens seit der von den Medien und der Politik im Jahr 2015 kolportierten „Flüchtlingskrise“, in der viele neue MigrantInnen nach Österreich gekommen sind, ist die Diskussion über den Sprachunterricht sowie die Sprachkenntnisse - in erster Linie der deutschen Sprache - wieder aufgeflammt und hat schließlich zu einer Reihe sprachenpolitischer Maßnahmen, darunter die Einführung sogenannter Deutschförderklassen, geführt. Die Sprachen der MigrantInnen selbst haben in der öffentlichen Diskussion allerdings kaum eine Rolle gespielt; ebenso wenig hat es eine Debatte über Mehrsprachigkeit gegeben.

Die Sprachenproblematik in der österreichischen Gesellschaft und insbesondere an Schulen ist sehr komplex und wird von politischen Parteien häufig als Mittel der Polarisierung benutzt. Nicht umsonst wählen Purkarthofer und Busch $(2013,8)$ die Bezeichnung ,umkämpftes Terrain“ hinsichtlich der Anerkennung und Wahrnehmung unterschiedlicher Herkunftssprachen von SchülerInnen und Lehrenden in den Schulen.

Diesem ,umkämpften Terrain“ widmet sich der vorliegende Artikel, indem er zunächst die sprachenpolitischen Bekenntnisse und Maßnahmen in Hinblick auf die Bildungssprache Deutsch sowie auf den mutter-, minderheiten- und fremdsprachlichen Unterricht in Österreich allgemein betrachtet und anschließend anhand einer Pilotstudie in Kärnten - dem österreichischen Bundesland an der Schwelle zur slawischen und romanischen Sprachgemeinschaft - untersucht, für welche (Fremd-)Sprachen sich die SchülerInnen interessieren und welche Gründe sie für ihr Interesse nennen.

\section{Sprachenpolitische Bekenntnisse}

Die Republik Österreich bekennt sich zur individuellen und gesellschaftlichen Mehrsprachigkeit. So hat Österreich die Europäische Charta der Regional- und Minderheitensprachen unterzeichnet, welche 2001 in Kraft getreten ist (vgl. Council of Europe 2020; Doleschal 2012) und den Schutz und die Förderung der Sprachen vorsieht, die auf dem österreichischen Staatsgebiet autochthon und gesetzlich anerkannt sind - Burgenlandkroatisch, Slowenisch, Slowakisch, Ungarisch, Tschechisch und Romanes.

Auch das Europäische Fremdsprachenzentrum (EFSZ), eine Einrichtung des Europarats, wo konkrete Maßnahmen zur praktischen Umsetzung sprachenpolitischer Empfehlungen des Europarats erarbeitet werden, um innovativen Sprachenunterricht zu fördern und die Mitgliedstaaten bei der Verbesserung der Sprachenbildung zu unterstützen, hat seinen Sitz in Österreich (Graz). Als nationale Kontaktstelle des EFSZ ist das Öster- 
reichische Sprachenzentrum (ÖSZ) im Auftrag des Bildungsministeriums eingerichtet, das die Aufgabe hat, SprachenexpertInnen und PraktikerInnen über die Aktivitäten des EFSZ zu informieren und seine Ergebnisse zu verbreiten.

Im Rahmen der Programme des Europarats und der Europäischen Union fördert Österreich das Erlernen von Sprachen, insbesondere von Fremdsprachen, in der schulischen Ausbildung. Dafür wurde ein Profil der Sprach- und Unterrichtspolitik (Language Education Policy Profile; kurz: LEPP) ausgearbeitet. Der sogenannte LEPP-Prozess (vgl. ÖSZ, BMUKK und BMBWF 2009) führte zur Erstellung des Curriculums Mehrsprachigkeit (Krumm und Reich 2011) sowie des Rahmenmodells Basiskompetenzen Spracbliche Bildungfür alle Lehrenden (Carré-Carlinger et al. 2014), welche auf die Nutzung aller sprachlichen Ressourcen von SchülerInnen abstellen und die Wertschätzung aller Sprachen zum Ziel haben, wobei jedoch Deutsch als Bildungssprache als zentrale Ressource angesehen wird.

Vor dem Hintergrund dieser sprachenpolitischen Bekenntnisse werden im Folgenden nun die Rolle und der Stellenwert der einzelnen Sprachen, das heißt, von Deutsch als Bildungssprache, nicht-deutscher Muttersprachen sowie von Fremdsprachen und autochthonen Minderheitensprachen, näher beleuchtet.

\subsection{Deutsch als Bildungssprache}

Wie eingangs erwähnt, stellt die Migration nach Österreich und in Verbindung damit die sprachliche Heterogenität der SchülerInnen das Bildungswesen vor große Herausforderungen, angesichts derer der Bildungserfolg und die Integration der zugezogenen Bevölkerung in die gesellschaftlichen und beruflichen Strukturen Österreichs zentral sind. So wird beispielsweise im 4. Staatenbericht zur Charta der Regional-und Minderheitensprachen (Bundeskanzleramt 2015, 13) festgehalten, dass ,allen SchülerInnen ungeachtet ihrer Herkunft und ihrer Sprache eine gute Schulbildung und Einstieg in die Arbeitswelt" ermöglicht werden sollen. Als Prämisse für den Bildungserfolg sowie die Integration in die Arbeitswelt und Gesellschaft wird die „gute Beherrschung der deutschen Sprache“ angeführt. Zur Sicherstellung der Beherrschung des Deutschen nennt der 4. Staatenbericht zur Charta der Regional- und Minderheitensprachen mehrere Maßnahmen wie zum Beispiel das verpflichtende Kindergartenjahr oder den speziellen Deutschförderunterricht. Neben der beruflichen und gesellschaftlichen Integration werden in diesem Bericht gute Kenntnisse der deutschen Sprache gleichsam als Voraussetzung für die „Verfestigung des Aufenthaltes“ von MigrantInnen sowie für den Erwerb der österreichischen Staatsbürgerschaft bestimmt.

Die Bildungssprache Deutsch steht ebenso auf der Webseite zur sprachlichen Bildung des Bundesministeriums für Bildung, Wissenschaft und Forschung (BMBWF 2020a) im 
Vordergrund. Wie auf der Webseite festgehalten wird, leiste die „Förderung der Unterrichtssprache Deutsch“" einen ,wesentliche[n] Beitrag zur Chancengleichheit“.

\subsection{Nicht-deutsche Muttersprachen und muttersprachlicher Unterricht}

Neben der Bildungs- und Unterrichtssprache Deutsch bekennt sich das BMBWF (2020a) auch zu allen anderen Sprachen außer Deutsch. So umfasse die sprachliche Bildung die "gesamte sprachliche Vielfalt“, ergo sowohl „Erst-, Zweit-, Herkunfts- und Fremdsprachen“ als auch die „Minderheitensprachen“. In Bezug auf diese sprachliche Vielfalt gibt es zahlreiche Förderungsmaßnahmen, darunter das Angebot zur Förderung der Erstsprachen eines/einer SchülerIn durch muttersprachlichen Unterricht.

Auch im 4. Staatenbericht zur Charta der Regional- und Minderheitensprachen wird festgehalten, dass großer Wert auf die Förderung und Entwicklung der „allenfalls nichtdeutsche[n] Muttersprache der Kinder" gelegt werde (Bundeskanzleramt 2015, 13). Dafür gebe es in Österreich ein „etabliertes System von muttersprachlichem Unterricht“, dessen Ziel der „Erwerb der Muttersprache zur Herstellung von Kontinuität und Stützung der Persönlichkeitsentwicklung" sei (Bundeskanzleramt 2015, 13f.). Betont wird außerdem, dass die „positive Einstellung zur Muttersprache und zum bikulturellen Prozess“ gefördert werden und die Kinder „[d]ie prinzipielle Gleichwertigkeit von Muttersprache und Deutsch im Unterricht" erleben sollen (Bundeskanzleramt 2015, 14).

Die Wichtigkeit der Anerkennung und Gleichwertigkeit verschiedener Sprachen entspricht auch den Plädoyers aus der Forschungsliteratur. So bekräftigt beispielsweise Busch (2016, 168) die Bedeutung der schulischen und gesellschaftlichen Akzeptanz der verschiedenen Herkunfts- und Muttersprachen der Kinder. Neben der Wertschätzung dieser Sprachen und ihrer Anerkennung ,als selbstverständliche, vollwertige Kommunikationssprachen" (ibid.) weist Rudolf de Cillia im Interview mit Beer $(2019,15)$ darauf hin, dass eine gute Beherrschung der Erst- bzw. Muttersprache positive Auswirkungen auf den Zweitsprachenerwerb habe. Ebenso erklärt Busch (2016, 168), dass sich beispielsweise das Verfassen von Texten in der Herkunfts- oder Muttersprache positiv auf die „Auseinandersetzung mit der deutschen Sprache" auswirke. Auch die soziale und identitätsbildende Funktion wird in Zusammenhang mit dem Unterricht in der Erst- bzw. Muttersprache häufig hervorgehoben (vgl. Tankır 2019, 13).

Trotz dieser wissenschaftlichen Erkenntnisse sowie der politischen Bekenntnisse in Bezug auf die Mutter- und Herkunftssprachen der SchülerInnen, ist der muttersprachliche Unterricht an österreichischen Schulen keine Selbstverständlichkeit. Das liegt zum einen daran, dass die Eltern und SchülerInnen das Recht auf diesen Unterricht selbst einfordern müssen, wofür sie jedoch auch das nötige Wissen über die dafür erforderlichen bürokratischen Wege benötigen. Daher wird das Recht auf den Unterricht in der Mut- 
tersprache häufig nicht in Anspruch genommen (vgl. Rienzner 2013, 35). Zum anderen kommt erschwerend hinzu, dass vielfach die festgelegte MindestschülerInnenzahl ${ }^{1}$ für den muttersprachlichen Sprachkurs nicht erfüllt werden kann.

\subsection{Fremdsprachen}

Dem Erlernen von Fremdsprachen kommt im österreichischen Bildungssystem eine besondere Bedeutung zu, was sich auch durch den LEPP-Prozess bestätigt hat (vgl. ÖSZ, BMUKK und BMBWF 2009). So beginnt der Erwerb der ersten lebenden Fremdsprache in Österreich bereits in der Primarstufe und wird durch ein „breite [s] Sprachlernangebot in der Sekundarstufe" fortgeführt (BMBWF 2020b).

Europaweit ist in Bezug auf die Fremdsprachenkenntnisse in der Europäischen Union der Barcelona-Beschluss des Europäischen Rates aus dem Jahr 2002 leitend. Dieser sieht vor, dass jede/r europäische BürgerIn zusätzlich zur eigenen Muttersprache noch zwei weitere Fremdsprachen beherrschen soll (vgl. Bundeskanzleramt 2015, 16), um die Mobilität der EU-BürgerInnen zu gewährleisten. Allerdings werde dieses ambitionierte Ziel in vielen EU-Staaten, darunter auch in Österreich, „noch nicht ausreichend erfüllt“ (Bundeskanzleramt 2015, 16). Wie das Spezial Eurobarometer 386 aus dem Jahr 2012 zeigt, wurde das Ziel der Beherrschung von mindestens zwei Fremdsprachen auf einem Niveau, um ein Gespräch führen zu können, in Österreich bisher zu 78\% lediglich für die erste Sprache erreicht (Europäische Kommission 2012, 18).

Dieses Ergebnis ist bei näherer Betrachtung der an österreichischen Schulen unterrichteten Fremdsprachen nicht verwunderlich: Laut einer Eurostat-Pressemitteilung (Grandovska und Rohner-Thielen 2017) haben in Österreich im Jahr 2015 nur 8,8\% der SchülerInnen an der Sekundarstufe I mehr als eine Fremdsprache gelernt. Dabei war Englisch mit 99,9\% die am häufigsten gelehrte Fremdsprache (vgl. ibid.). Das Faktum, dass in Österreich nach wie vor Englisch „mit weitem Abstand zu anderen Sprachen“ die am häufigsten unterrichtete Fremdsprache darstellt, wird auch im 4. Staatenbericht zur Charta der Regional-und Minderheitensprachen konstatiert (vgl. Bundeskanzleramt 2015, 16).

Die Dominanz von Englisch als Fremdsprache im österreichischen Schulsystem wird auch in den jährlichen Statistiken ersichtlich. So illustrieren die Daten der Statistik Austria (2019) für das Schuljahr 2017/18, dass Englisch auf allen Schulstufen und in allen Schultypen mit 97,8\% deutlich überwiegt. Die romanischen Sprachen Französisch (7,6\%), Italienisch (4,9\%), Spanisch (4,5\%) sind demgegenüber weit abgeschlagen und an fünfter Stelle folgt schließlich Russisch (0,7\%). Latein hat einen Anteil von 5,2\%, auf

1 An allgemeinbildenden höheren Schulen (AHS) müssen mindestens zwölf Personen pro Sprache angemeldet sein und an allgemeinbildenden Pflichtschulen je nach Bundesland fünf bis zwölf TeilnehmerInnen (vgl. Rienzner 2013, 35). 
andere lebende Fremdsprachen entfallen zusammen rund 1,4\%. Es handelt sich dabei um folgende Sprachen, die im Lehrplan der Neuen Mittelschulen (NMS) und der allgemeinbildenden höheren Schulen (AHS) taxativ aufgezählt werden: Bosnisch/Kroatisch/Serbisch, Kroatisch, Polnisch, Slowenisch, Slowakisch, Tschechisch und Ungarisch. Türkisch wird nur im Lehrplan der NMS angeführt (vgl. RIS 2020a; RIS 2020b).

Die Diskrepanz zwischen politischen Bekenntnissen in Bezug auf die Beherrschung mehrerer Fremdsprachen und der praktischen Umsetzung lässt sich auch anhand der Daten zur Mobilität der SchülerInnen nachzeichnen: So spricht sich zwar das BMBWF (2020b) auf seiner Webseite dezidiert für eine europaweite und internationale Unterstützung von Schulprojekten aus und erklärt, dass „Kontakte und Aufenthalte im Ausland, zum Beispiel über das Erasmus+-Programm, [...] häufig ganz automatisch die Vorzüge der Mehrsprachigkeit bewusst [machen] und [...] Schüler/innen [motivieren] andere Sprachen zu lernen." Die OeAD-Daten des Mobilitätsradars Bildung 2017 (OeAD 2018, 15) zeigen jedoch, dass der überwiegende Teil schulischer Auslandsaufenthalte mit ERASMUS + in englischsprachige Länder (Vereinigtes Königreich und Irland) sowie Deutschland führt. Mit dieser starken Präferenz von Auslandsaufenthalten der SchülerInnen in englisch- und deutschsprachigen Ländern wird allerdings das vom BMBWF deklarierte Ziel der Förderung und Bewusstmachung von Mehrsprachigkeit klar verfehlt.

Die vorliegenden Zahlen illustrieren, dass die sprachenpolitischen Bekenntnisse in Bezug auf die Mehrsprachigkeit der Bevölkerung in Österreich zwar in Form von Beschlüssen festgehalten sind; die tatsächliche Implementierung ist jedoch ernüchternd bzw. wird nicht forciert, da ansonsten eine derartige Dominanz von Englisch über alle anderen Fremdsprachen in Österreich nicht erklärbar wäre.

Die Disproportionalität zwischen den in den Schulen unterrichteten Sprachen - wobei nach Englisch drei romanische Sprachen (Französisch, Italienisch, Spanisch) folgen und alle anderen Sprachen zusammen nicht einmal 1,5\% ausmachen - ist auch der Bewertung der einzelnen Sprachen geschuldet. Hierbei handelt es sich um ein Phänomen der longue durée, denn schon in der K.-u.-k.-Monarchie gab es die Kategorie der „Cultursprachen“ (Pederin 1995, 94; Wolf 2012, 170), zu denen jedenfalls die slawischen Sprachen der Monarchie nicht zählten, wohl aber beispielsweise Italienisch. In diesem Zusammenhang sprechen Aygün-Sagdic, Bajenaru und Melter $(2015,112)$ auch von der „Hegemonie der Kolonialsprachen“, da im Bildungssystem bestimmte Sprachen ein höheres Prestige besitzen würden als andere Sprachen:

Bestimmte Natiolekte werden als ,Fremdsprachen' im Bildungssystem gelehrt, gelernt und wertgeschätzt, andere abgewertet und verboten. Typischerweise sind es europäische Kolonialsprachen wie Englisch, Französisch, 
Deutsch und Spanisch, die in europäischen Bildungssystemen besondere Wertschätzung erfahren.

Wie Krumm $(2011,104)$ festhält, wäre es jedoch „[a] us sprachpädagogischer und sprachenpolitischer Sicht" sinnvoll, wenn die erste gelernte Fremdsprache aus dem Lebensumfeld der SchülerInnen komme. Dazu zählen zum Beispiel die Sprache eines Nachbarlandes oder ortsansässiger Minderheiten.

\subsection{Autochthone Minderheitensprachen}

Wie bereits erwähnt, sind in Österreich die Minderheitensprachen Burgenlandkroatisch, Slowenisch, Slowakisch, Ungarisch, Tschechisch, Romanes sowie die Österreichische Gebärdensprache gesetzlich und verfassungsmäßig anerkannt. ${ }^{2}$ Bei der letzten Volkszählung mit Erhebung der „Umgangssprache“ im Jahr 2001 gaben lediglich 1,5\% der Wohnbevölkerung in Österreich an, eine der offiziell anerkannten autochthonen Minderheitensprachen als Umgangssprache zu verwenden (vgl. De Cillia 2020, 82). ${ }^{3}$ Wie De Cillia und Wodak $(2006,44)$ festhalten, ist seit 1945 ein „drastischer Rückgang“ bei den SprecherInnen autochthoner Minderheitensprachen zu beobachten. Mehrsprachigkeit, so das Fazit der AutorInnen, existiere in Österreich daher hauptsächlich aufgrund der Sprachen der MigrantInnen (vgl. ibid.).

Trotz der rückläufigen Zahl an SprecherInnen autochthoner Minderheitensprachen gibt es aufgrund verfassungsrechtlicher Bestimmungen in Österreich ein auf zweisprachiger Bildung basierendes Minderheitenschulwesen wie beispielsweise in Kärnten - dem Bundesland, in dem auch die vorliegende Pilotstudie durchgeführt wurde.

\section{Ausgangssituation: Bundesland Kärnten}

Das Bundesland Kärnten ist von "gesellschaftlicher Mehrsprachigkeit“ geprägt, da „auf ein- und demselben Territorium mehrere Sprachen gesprochen werden" (Riehl 2013, 378): österreichisches Deutsch sowie das Kärntner Slowenische, das eine Vielzahl an verschiedenen Varietäten des gesprochenen Slowenischen umfasst (vgl. Busch 2010, 177). Das Prestige der slowenischen Sprache hat sich in Kärnten in den letzten Jahren in eine positive Richtung verändert, da Slowenisch mit der Eigenstaatlichkeit Sloweniens zu einer Staatssprache avanciert ist (vgl. ibid., 179). Seit dem Beitritt Sloweniens zur Eu-

\footnotetext{
2 Zudem gibt es noch weitere neue, jedoch nicht anerkannte Minderheitensprachen wie zum Beispiel Bosnisch/Kroatisch/Serbisch/Montenegrinisch, Türkisch oder Kurdisch (vgl. De Cillia 2020, 81).

3 Die Anzahl der SprecherInnen der Österreichischen Gebärdensprache, die mit circa 10000 beziffert wird, wurde 2001 nicht erhoben (vgl. De Cillia 2020, 83).
} 
ropäischen Union (EU) im Jahr 2004 wurde Slowenisch zudem eine Amtssprache der EU.

In Bezug auf die Schulsprachenpolitik existieren für die slowenische Volksgruppe in Kärnten sowie für die kroatische und ungarische Volksgruppe im Burgenland eigene Schulgesetze (vgl. De Cillia 2020, 89). Diese Gesetze sehen unter anderem einen bilingualen Unterricht in der Volksschule vor. Nach der Volksschule kann die jeweilige Minderheitensprache in den Schulen beispielsweise als Wahlpflichtgegenstand besucht werden. Eine Ausnahme bieten die durchgehend bilingualen Schulen wie das Bundesgymnasium/Bundesrealgymnasium (BG/BRG) für Slowenen, die zweisprachige Handelsakademie in Klagenfurt/Celovec und die zweisprachige HLW in St. Peter im Rosental/Šentjakob v Rožu.

Die Durchsetzung der Gesetze und Sprachenrechte für autochthone Minderheiten, die im Staatsvertrag von Wien verankert sind, wurde jedoch von politischer Seite immer wieder behindert und musste häufig durch Entscheidungen des Verfassungsgerichtshofs eingefordert werden. Beispiele dafür sind der zweisprachige Unterricht auch in der vierten Klasse Volksschule sowie die zweisprachigen Ortstafeln in Kärnten (vgl. De Cillia 2020, 89f.).

Neben Deutsch und Slowenisch sind in Kärnten aufgrund seiner topographischen Lage im Alpen-Adria-Raum auch Italienisch sowie die Sprachen aus dem ex-jugoslawischen Raum, insbesondere Kroatisch, von großer kultureller und gesellschaftlicher Relevanz. Aus linguistischer Perspektive ist Kärnten vor allem deshalb interessant, weil hier drei Sprachen unterschiedlicher indoeuropäischer Sprachfamilien - germanisch, romanisch und slawisch - aufeinandertreffen. Aufgrund der räumlichen Nähe zu Italien, Slowenien und Kroatien sowie der Existenz einer autochthonen Minderheit wären für das Bundesland Kärnten die Verwirklichung der Empfehlungen Krumms (2011, 104), dass die SchülerInnen Fremdsprachen aus ihrem Lebensumfeld lernen sollten, besonders sinnvoll. Auch im Hinblick auf den Barcelona-Beschluss des Europäischen Rates, dessen Ziel die Forcierung der Kommunikation, des Austausches und der Annäherung der europäischen Mitgliedsländer sind, würde es sich an Kärntner Schulen anbieten, diese Sprachen der Nachbarländer bzw. der Alpen-Adria-Region zu lernen. ${ }^{4}$

Wie in Abschnitt 1.3 dargestellt wurde, sind die slawischen Sprachen im Fremdsprachenunterricht an österreichischen Schulen sehr spärlich vertreten. Im Zuge der geplanten Wiedereinführung des Lehramtsstudiums von Russisch und Bosnisch/Kroatisch/

4 Diesbezüglich gibt es hier bereits richtungsweisende Projekte wie die sogenannten „Kugy-Klassen“ des BG/BRG für Slowenen (https://www.slog.at/schule/detail/C13/kugy-weil-mehrsprachigkeit-eingeschenk-ist-perche-il-multilinguismo-e-un-d). 
Serbisch $(\mathrm{B} / \mathrm{K} / \mathrm{S})^{5}$ an der Alpen-Adria-Universität Klagenfurt, wurde daher zunächst vom Institut für Slawistik eine Interessenserhebung unter SchuldirektorInnen an Kärntner Schulen durchgeführt, um zu eruieren, ob die Schulen Interesse an einer Erweiterung ihres Fremdsprachenangebotes um diese beiden Unterrichtsfächer haben. Daran anschließend wurde eine Pilotstudie unter SchülerInnen getätigt, um herauszufinden, welche sprachlichen Interessen die jungen Menschen haben, die den schulischen Sprachunterricht besuchen. Zu diesem Zweck wurde ein Fragebogen entwickelt, mithilfe dessen Schulkinder verschiedenster Alters- und Schulstufen nach ihrem persönlichen Interesse und ihren Wünschen in Bezug auf die an Kärntner Schulen unterrichteten (Fremd)Sprachen befragt wurden. Für diese Untersuchung wurden folgende zwei Forschungsfragen erstellt:

(1) Für welche (Fremd-)Sprachen interessieren sich die SchülerInnen an Kärntner Schulen?

(2) Welche (Fremd-)Sprachen wollen die SchülerInnen lernen und welche Gründe sind für ihre Wahl ausschlaggebend?

Die Ergebnisse der Interessenserhebung unter SchuldirektorInnen sowie der anschließenden Fragebogenerhebung unter SchülerInnen an Kärntner Schulen werden in den nächsten Abschnitten präsentiert.

\section{Untersuchungsmethoden}

Am Beginn der vorliegenden Pilotstudie stand zunächst das Desiderat herauszufinden, ob in Schulen, in denen Russisch und Bosnisch/Kroatisch/Serbisch (B/K/S) kein Unterrichtsfach ist, ein Interesse besteht, den schulischen Fremdsprachenunterricht um diese beiden slawischen Sprachen zu erweitern. Die Interessenserhebung wurde zunächst unter SchuldirektorInnen von allgemein- und berufsbildenden höheren Schulen Kärntens durchgeführt und schließlich zu einer Untersuchung unter SchülerInnen ausgeweitet, um deren Präferenzen bezüglich des Erlernens von (Fremd-)Sprachen zu eruieren. Diese zwei Schritte sowie die Methode der Fragebogenerhebung ${ }^{6}$ werden in den nächsten beiden Abschnitten im Detail vorgestellt.

\footnotetext{
$5 \mathrm{~B} / \mathrm{K} / \mathrm{S}$ hat in der deutschsprachigen Slawistik die seit dem 19. Jahrhundert gängige Bezeichnung „Serbokroatisch“" abgelöst, da die betroffenen Völker ihre Standardsprachen seit dem Zerfall Jugoslawiens unabhängig entwickeln und normieren. Es existiert auch das Akronym B/K/M/S, welches das später gesetzlich festgelegte Montenegrinische einbezieht. Aus linguistischer Sicht handelt es sich bei B/K/S um eine plurizentrische Sprache im Sinne Ammons (vgl. Bunčić 2008).

${ }^{6}$ Diese Erhebung wurde von Sandra Husanović und Joulia Köstenbaumer durchgeführt.
} 


\subsection{Interessenserhebung unter SchuldirektorInnen}

Im Zuge der geplanten Wiedereinführung der Lehramtsstudien Russisch sowie Bosnisch/ Kroatisch/Serbisch am Institut für Slawistik der Alpen-Adria-Universität Klagenfurt wurde im Februar 2019 mit Unterstützung der Bildungsdirektion für Kärnten eine Interessenserhebung an alle DirektorInnen allgemein- und berufsbildender höherer Schulen (AHS, BAfEP, HAK, HUM, HTL) Kärntens ausgeschickt. In dieser per E-Mail versendeten Interessenserhebung wurden die DirektorInnen gefragt, ob sie die Möglichkeit einer Erweiterung des Fremdsprachenangebotes ${ }^{7}$ an ihrer Schule um die Sprachen Russisch und/oder Bosnisch/Kroatisch/Serbisch begrüßen würden, wobei als Antwortmöglichkeiten die beiden Optionen „eher ja“ oder „eher nein“ zur Auswahl standen.

Von den insgesamt 51 allgemein- und berufsbildenden höheren Schulen Kärntens ${ }^{8}$ haben 18 Schulen von sieben unterschiedlichen Standorten9 Anfang März 2019 auf die Interessenserhebung geantwortet. Das bedeutet, dass weniger als die Hälfte aller kontaktierten Schulen, nämlich lediglich rund 35\%, auf die Umfrage reagiert haben, wobei diese geringe Rücklaufquote bei schriftlichen Befragungen üblich ist (vgl. Diekmann 2014, 516).

Trotzdem skizzieren die Ergebnisse dieser Interessenserhebung eine erste Tendenz, die in Bezug auf den Fremdsprachenunterricht an Kärntner allgemein- und berufsbildenden höheren Schulen bemerkenswert ist: Exakt ein Drittel der DirektorInnen, die eine Rückmeldung gegeben haben, würden die Möglichkeit einer Erweiterung des Fremdsprachenangebotes an ihrer Schule um die Sprachen Russisch und B/K/S begrüßen. Auffällig ist, dass die SchulleiterInnen, die diese Erweiterung befürworten würden, immer beide Sprachen ausgewählt haben. Lediglich eine Schule gab an, dass sie Russisch schon anbiete, der Einführung eines Fremdsprachenunterrichts in $\mathrm{B} / \mathrm{K} / \mathrm{S}$ jedoch nicht zustimmen würde.

Als Gründe für die grundsätzliche Bereitschaft, einen Fremdsprachenunterricht in diesen beiden slawischen Sprachen einzuführen, gaben die DirektorInnen an, dass sie diese als „wertvolle Zusatzqualifikation“ sehen und „jede zusätzliche Fremdsprache ein Gewinn" sei. Allerdings seien bei einer Einführung eines neuen Unterrichtsfaches zusätzliche finanzielle und personelle Ressourcen zu bedenken.

Die Direktion einer Schule, die zwar in der Interessenserhebung mit „eher nein“ für Russisch und B/K/S geantwortet hatte, fügte bei der Begründung hinzu, dass von den sla-

\footnotetext{
7 Es wurde in der Umfrage ausdrücklich darauf hingewiesen, dass es sich um Fremdsprachenunterricht und nicht um muttersprachlichen Unterricht handelt.

8 Die Anzahl der Schulen pro Schultyp ist auf der Homepage der Bildungsdirektion für Kärnten https://info.bildung-ktn.gv.at/schoolGuide abrufbar.

9 Die Standorte dieser Schulen sind: Althofen, Feldkirchen, Ferlach, Hermagor, Klagenfurt, St. Jakob im Rosental sowie Villach.
} 
wischen Sprachen Slowenisch aufgrund der Grenznähe eine „interessante Option“ darstellen würde.

Zwei Drittel der SchuldirektorInnen haben negativ auf die Interessenserhebung geantwortet. Als Begründung für die Ablehnung wurde beispielsweise auf Englisch als sprachlichen Schwerpunkt oder auf die niedrige Nachfrage bzw. das geringe Interesse verwiesen, welche die SchülerInnen für die slawischen Sprachen zeigen würden.

Nach der Entwicklung und der Übermittlung der Interessenserhebung an die SchuldirektorInnen wurde ein eigener Fragebogen erstellt, um herauszufinden, welche Sprachlernpräferenzen diejenigen haben, die von den Entscheidungen der DirektorInnen direkt betroffen sind: die Schülerinnen und Schüler. Ziel dieses Fragebogens war es allgemein zu ermitteln, welche (Fremd-)Sprachen die SchülerInnen bereits lernen und welche sie gerne lernen würden. Aus diesem Grund wurde die Untersuchung nicht auf die slawischen Sprachen Russisch und Bosnisch/Kroatisch/Serbisch beschränkt, sondern bei der Ausarbeitung des Fragebogens wurde darauf geachtet, allgemeine und sprachunspezifische Fragen zu stellen, um ein möglichst breites Spektrum an Sprachen zu erfassen. Die Beschreibung der Fragebogenerstellung folgt im nächsten Abschnitt.

\subsection{Entwicklung des SchülerInnenfragebogens}

Im Anschluss an die Interessenserhebung unter SchuldirektorInnen stellte sich unmittelbar die Frage, welche Präferenzen diejenigen, die den Sprachunterricht in der Schule besuchen, in Bezug auf verschiedene Sprachen haben. Daher wurde ausgehend von den zwei Forschungsfragen, (1) für welche (Fremd-)Sprachen sich SchülerInnen an allgemein- und berufsbildenden höheren Schulen interessieren und (2) welche (Fremd-)Sprachen sie aus welchen Gründen gerne lernen würden, ein Fragebogen entwickelt.

Die Entscheidung, die Umfrage unter SchülerInnen mithilfe eines schriftlichen Fragebogens durchzuführen, resultierte erstens in dessen klarem Vorteil gegenüber mündlichen Befragungen wie beispielsweise Interviews, da bei einem Fragebogen in relativ kurzer Zeit eine große Anzahl von Personen befragt werden kann (vgl. Atteslander 2010, 157). Zweitens erfasst ein Fragebogen vor allem quantitative Aspekte und daher eignete sich dieser Befragungstyp besonders für die vorliegende Studie, da sie vor allem herausfinden wollte, wie viele SchülerInnen welche und wie viele verschiedene (Fremd-)Sprachen lernen bzw. lernen wollen. Drittens eignet sich ein Fragebogen speziell für die Befragung von größeren Gruppen wie Schulklassen (vgl. Diekmann 2014, 516).

Um den drei Gütekriterien der Objektivität, Reliabilität und Validität gerecht zu werden, ist bei der Entwicklung von Fragebögen besonders auf die Formulierung der Fragen und den Fragetyp - eher geschlossen oder eher offen - zu achten. Der Vorteil von geschlossenen Fragen liegt einerseits in ihrer einfachen Auswertbarkeit, der größeren Ein- 
heitlichkeit und somit in der erhöhten Vergleichbarkeit der einzelnen Antworten (vgl. Atteslander 2010, 148). Dadurch verbessern sich auch die Objektivität und die Reliabilität der Befragung. Andererseits werden bei geschlossenen Fragen keine „Informationen jenseits des Spektrums der vorgelegten Antwortkategorien“ (Diekmann 2014, 438) gegeben und zudem besteht die Gefahr der „Suggestivwirkung“" (Atteslander 2010, 148). Ein nicht unerheblicher Nachteil offener Fragen liegt hingegen darin, dass diese häufig von den Befragten nicht beantwortet werden (vgl. Atteslander 2010, 148). Ein Kompromiss besteht laut Diekmann $(2014,478)$ daher in der Formulierung sogenannter „Hybridfragen“, welche neben einer geschlossenen Antwortkategorie zusätzlich eine offene Antwortmöglichkeit bieten.

Neben der Wahl des geschlossenen oder offenen Fragetyps ist die Formulierung der einzelnen Fragen wichtig, da diese verständlich, konkret, neutral und eindeutig gestellt sein müssen (vgl. Diekmann 2014, 479-482). Insbesondere die Verständlichkeit und Eindeutigkeit spielten bei dem Fragebogen der vorliegenden Untersuchung eine wichtige Rolle, da die ForscherInnen während der Beantwortung der Fragebögen in der jeweiligen Schule nicht anwesend und daher auch keine Verständnisfragen seitens der SchülerInnen möglich waren.

Für die Umfrage unter SchülerInnen wurde von den Forscherinnen im Dreierteam ${ }^{10}$ Ende Februar 2019 ein erster Fragebogen entwickelt, der zwei offene und drei geschlossene Fragen enthielt. Dieser Fragebogen wurde im Rahmen der Veranstaltung „Schule Meets Uni“, die an der Universität Klagenfurt am 26.02.2019 stattgefunden hat, an insgesamt 70 SchülerInnen der Neuen Mittelschule der Pädagogischen Hochschule Kärnten getestet. Mithilfe dieses Pretests stellte sich heraus, dass eine Frage zu kompliziert und zu lang und zwei andere Fragen zu unspezifisch bzw. zu undifferenziert waren. Daher wurde der Fragebogen im Anschluss an den Pretest noch einmal grundlegend überarbeitet, indem der Fokus auf die Konkretheit und Verständlichkeit der Frageformulierung gelegt wurde. Außerdem wurde nur eine geschlossene Frage beibehalten, während die beiden anderen geschlossenen Fragen in offene umgewandelt wurden, um den SchülerInnen die Möglichkeit zu geben, mehrere Sprachen zu nennen. Die überarbeitete Version wurde noch einmal an fünf Kinder von KollegInnen verteilt, die eine allgemein- bzw. berufsbildende höhere Schule in Kärnten besuchen, und diese wurden gebeten, die Fragen auf deren Eindeutigkeit und Verständlichkeit zu beurteilen. Nach diesem weiteren Pretest wurden nur mehr kleinere Änderungen am Fragebogen vorgenommen und die finale Version erstellt (Abb. 1).

\footnotetext{
${ }^{10}$ Der Fragebogen wurde von Sandra Husanović und Joulia Köstenbaumer in Zusammenarbeit mit Magdalena Kaltseis erstellt und verteilt.
} 


\section{Fragebogen Mehrsprachigkeit \\ Alter: \\ Geschlecht: o männlich o weiblich \\ Muttersprache(n):}

Frage 1: Welche Sprachen lernst du in der Schule? Zähle sie bitte auf.

Frage 2: Welche Sprachen würdest du gerne lernen, die es an deiner Schule nicht gibt? Zähle auf und erkläre bitte kurz, warum du diese Sprachen gerne lernen würdest.

Frage 3: Lernst du bereits eine dieser Sprachen, die du bei Frage 2 genannt hast, außerhalb der Schule? Wenn ja, wo und welche Sprache?

Frage 4: Welchen Fremdsprachenunterricht sollte man deiner Meinung nach an Kärntner Schulen besuchen können? Kreuze an.
o Arabisch
o Griechisch
o Serbisch
o Bosnisch
o Italienisch
o Slowenisch
o Chinesisch
o Japanisch
o Spanisch
o Englisch
o Kroatisch
o Türkisch
o Französisch
o Russisch
o Andere

Frage 5: Wenn du die Möglichkeit hättest, die Muttersprache eines Mitschülers/einer Mitschülerin zu lernen, welche Sprache(n) wäre(n) das?

Abbildung 1: Fragebogen Mehrsprachigkeit.

Wie aus diesem Fragebogen (Abb.1) hervorgeht, wurden die Fragen in der pronominalen Du-Form-Anrede gestellt, weil diese der üblichen Anredeform von Kindern und Jugendlichen, die noch nicht volljährig sind, entspricht.

\subsection{Untersuchungsgruppe}

Für die Fragebogenerhebung wurden die SchülerInnen derjenigen Schulen ausgewählt, an denen die DirektorInnen die Möglichkeit einer Erweiterung des bisherigen Fremdsprachenangebots an ihrer Schule um Russisch sowie Bosnisch/Kroatisch/Serbisch positiv beantwortet hatten. ${ }^{11}$ Rückblickend wäre wahrscheinlich eine Untersuchung in den Schulen, die in ihrer Rückmeldung auf das mangelnde Interesse der SchülerInnen an

\footnotetext{
${ }^{11}$ Zwei Gymnasien, welche die Interessenserhebung in beiden Sprachen mit „eher ja“ beantwortet hatten, haben sich bezüglich der Bitte um die Durchführung einer Fragebogenerhebung unter SchülerInnen nicht zurückgemeldet. Außerdem wurde die Fragebogenerhebung auch in der Schule durchgeführt, in der Russisch als Fremdsprache bereits angeboten wird, die jedoch die Einführung eines Fremdsprachenunterrichts in $\mathrm{B} / \mathrm{K} / \mathrm{S}$ nicht begrüßen würde.
} 
slawischen Sprachen verwiesen haben, ebenso lohnend und interessant gewesen, um zu überprüfen, ob die Einschätzung der DirektorInnen mit den Interessen und Wünschen der SchülerInnen korreliert. Diese Umfrage soll daher zu einem anderen Zeitpunkt nachgeholt werden.

Als Untersuchungsgruppe wurden die SchülerInnen der ersten und zweiten Klassen der Sekundarstufe I sowie der ersten und zweiten Klassen der Sekundarstufe II allgemeinund berufsbildender höherer Schulen festgelegt. Der Grund für die Auswahl der SchülerInnen dieser Schulstufen bestand darin, dass in der ersten und zweiten Klasse der Sekundarstufe I sowie in den ersten beiden Klassen der Sekundarstufe II die SchülerInnen häufig vor der Wahlmöglichkeit einer weiteren (lebenden) Fremdsprache stehen. Während in vielen allgemein- und berufsbildenden höheren Schulen Englisch bereits ab der ersten Klasse unterrichtet wird, stehen ab der dritten Schulklasse der Sekundarstufe I sowie der dritten Klasse der Sekundarstufe II meist weitere Fremdsprachen, vor allem die drei romanischen Sprachen Französisch, Italienisch oder Spanisch, zur Auswahl.

Nach offizieller Genehmigung durch die Bildungsdirektion für Kärnten wurde die Fragebogenerhebung schließlich an fünf Schulen (zwei Gymnasien, einem Oberstufengymnasium, einer Handelsakademie und einer Höhere Bundeslehranstalt für wirtschaftliche Berufe) durchgeführt. Davon sind zwei Schulen (eine Handelsakademie und eine Höhere Bundeslehranstalt für wirtschaftliche Berufe) zweisprachig (Slowenisch und Deutsch) geführt, weshalb die Ergebnisse der Fragebogenerhebung in der Sekundarstufe II immer in Hinblick auf diesen besonderen Umstand zu interpretieren sind.

Insgesamt haben 475 SchülerInnen der Sekundarstufe I und 338 SchülerInnen der Sekundarstufe II an der Fragebogenerhebung teilgenommen. 41,5\% der TeilnehmerInnen der Sekundarstufe I waren weiblich und 57,9\% männlich. 0,6\% der Befragten haben keine Angabe zum Geschlecht gemacht. Im Durchschnitt waren die SchülerInnen der Sekundarstufe I 11,1 Jahre alt. In der Sekundarstufe II waren 56,6\% der Befragten weiblich und 42,3\% männlich. 0,9\% haben keine Angabe zum Geschlecht gemacht. Das durchschnittliche Alter der TeilnehmerInnen der Sekundarstufe II betrug 15,7 Jahre.

\section{Ergebnisse der Fragebogenauswertung}

Die Antworten auf die Fragen, welche die SchülerInnen handschriftlich beantwortet hatten, wurden in eine Excel-Tabelle exportiert und anschließend statistisch ausgewertet. Die Inhalte der Antworten auf die zweite (F2) sowie die dritte Frage (F3), welche neben den einzelnen Sprachen Gründe für das Erlernen einer Sprache sowie außerschulische Sprachlernorte enthielten, wurden zusätzlich inhaltsanalytisch zusammengefasst, um die wesentlichen Inhalte zu erfassen. 
Zur besseren Übersicht werden die Ergebnisse der Fragebogenauswertung ${ }^{12}$ darstufe I sowie der Sekundarstufe II getrennt dargestellt. Am Ende der Ergebnispräsentation werden die wichtigsten Ergebnisse der beiden Auswertungen miteinander verglichen.

\subsection{Ergebnisse: Sekundarstufe I}

Von den insgesamt 475 befragten SchülerInnen der Sekundarstufe I haben 88,8\% angegeben, dass ihre Muttersprache Deutsch ist. 4,4\% der Befragten haben keine Angabe zur Muttersprache gemacht. ${ }^{13}$ Nach Deutsch sind die am öftesten von den TeilnehmerInnen der Untersuchung genannten Muttersprachen „Kroatisch“ (1,7\%), „Bosnisch“ (1,5\%) und „Englisch“ (1,1\%). Außerdem führten einige SchülerInnen „Französisch“ $(0,8 \%)$, „Russisch“ (0,8\%), „Spanisch“ (0,6\%), „Ungarisch“ (0,6\%) sowie „Rumänisch“ (0,6\%) als ihre Muttersprachen an. Weitere von den SchülerInnen genannte Muttersprachen (< 0,5\%) sind „Albanisch“, „Bulgarisch“, „Chinesisch“, „Niederländisch“, „Schwedisch“, „Serbokroatisch“, „Österreichisch“ und „Türkisch“.

Die Auswertung der unterschiedlichen Muttersprachen der SchülerInnen ist aus mehreren Gründen aufschlussreich. Erstens ist auffällig, dass im Bundesland Kärnten, das an Italien und Slowenien grenzt, in der Sekundarstufe I kein/e SchülerIn als Muttersprache eine dieser beiden Sprachen, die auch im öffentlichen Leben des Landes präsent sind (Supermärkte, Hinweisschilder, Plakate etc.), angegeben hat. Zweitens fällt sofort auf, dass die Gruppe derjenigen, die als Muttersprache „Deutsch“ notiert haben, mit 88,8\% sehr hoch ist. Demnach haben nur 6,8\% ${ }^{14}$ der SchülerInnen der Sekundarstufe I eine andere Muttersprache als Deutsch oder neben Deutsch noch eine zweite Sprache als Muttersprache $(3,8 \%)$ angegeben. Dieses Ergebnis deckt sich nicht mit dem von der Statistik Austria für das Jahr 2017/18 herausgegeben Informationsblatt über den muttersprachlichen Unterricht in Österreich, in dem für das Bundesland Kärnten die Zahl der SchülerInnen mit anderer Muttersprache als Deutsch für die AHS mit 11,4\% beziffert ist (vgl. Garnitschnig 2019, 6). Ein möglicher Grund für die hohe Anzahl derer, die Deutsch als Muttersprache genannt haben, könnte sein, dass sie in diesem Feld die Bildungs- und

\footnotetext{
${ }^{12}$ Die Autorinnen danken Gerhild Gram für die große Unterstützung bei der Auswertung.

${ }^{13}$ Die Autorinnen des Fragebogens haben sich für den häufig verwendeten Begriff der „Muttersprache“ entschieden, da er in der Umgangssprache „als etwas klar Verständliches“ gilt (Corazza 2016, 3). Wir sind uns bewusst, dass der Terminus auch ideologische Konnotationen hat. Bei einer erneuten Fragebogenerhebung wäre es daher eine Überlegung wert, den Begriff zu umschreiben, etwa mit Phrasen wie „Zuhause spreche ich ..." / „Mit meinen Freunden spreche ich ...“ / „In der Schule spreche ich .... Auf diese Weise wäre auch eine differenziertere Darstellung des Sprachgebrauchs der Kinder und Jugendlichen in verschiedenen Lebensbereichen möglich.

${ }^{14}$ Die Zahl ist die Differenz von 100\% und 93,2\%. Letztere Prozentangabe entstand aus der Addition der Prozentzahl, die Deutsch als Muttersprache angegeben haben $(88,8 \%)$, und derjenigen, die keine Angabe gemacht haben $(4,4 \%)$.
} 
Zielsprache Deutsch angegeben haben, um einer gesellschaftlichen und politischen Erwartungshaltung zu entsprechen. ${ }^{15}$

Des Weiteren ist bemerkenswert, dass nach Deutsch die meisten der befragten SchülerInnen Bosnisch/Kroatisch/Serbisch (3,6\%) als Muttersprache angeben. Das erklärt sich daraus, dass in den letzten Jahrzehnten eine große Anzahl an Menschen aus dem ehemaligen Jugoslawien nach Österreich migriert ist. So gehörten die SprecherInnen aus dem ehemaligen Jugoslawien beispielsweise bereits in der Volkszählung aus dem Jahr 2001 zur größten Sprachgruppe nach Deutsch (vgl. De Cillia und Wodak 2006, 20). Die SchülerInnen nennen ihre Muttersprachen „Bosnisch“, „Kroatisch“ und in einem Fall „Serbokroatisch“. Wir fassen diese Nennungen als B/K/S zusammen, wie es in der deutschsprachigen Slawistik üblich ist. Außerdem zeigt das Ergebnis der Auswertung der Muttersprachen der SchülerInnen eindeutig, dass die SchülerInnen mehrsprachig sind - alleine bei den Muttersprachen wurden 16 Sprachen genannt, darunter auch von zwei Personen „Österreichisch“.

Auf die erste Frage (F1), welche Sprachen sie in der Schule lernen, haben die SchülerInnen der ersten und zweiten Klasse der Sekundarstufe I vor allem „Englisch“ (98,3\%) und „Deutsch“ $(64,4 \%)$ genannt, was mit der Stundentafel vieler Gymnasien übereinstimmt, da Englisch meistens ab der ersten und eine weitere (lebende) Fremdsprache erst ab der dritten Klasse Gymnasium unterrichtet werden. Interessant ist, dass Deutsch lediglich von zwei Drittel der SchülerInnen als Antwort auf F1 gegeben wurde, obwohl Deutsch an allen untersuchten Schulen unterrichtet wird und es sich hier ebenso um einen Sprachunterricht handelt. Einige SchülerInnen haben außerdem angegeben, dass sie in der Schule „Italienisch“ (17,1\%) sowie „Französisch“ (4,4\%) lernen. Ein Blick auf die Stundentafel der beiden Gymnasien, in denen die Untersuchung durchgeführt wurde, zeigt jedoch, dass Italienisch und Französisch erst ab der dritten Klasse der Sekundarstufe I in der Stundentafel angeführt sind. Es könnte daher sein, dass manche SchülerInnen eine dieser Sprachen bei F1 angegeben haben, weil sie bereits wissen, dass sie im nächsten Schuljahr diese Sprache lernen werden bzw. wollen.

Die zweite Frage (F2) der Fragebogenerhebung, welche Sprachen die SchülerInnen gerne lernen würden, die es an der Schule nicht gibt, wurde bewusst offen gestellt, um den SchülerInnen die Möglichkeit zu geben, selbstständig mehrere Sprachen zu nennen. Insgesamt haben die SchülerInnen 31 verschiedene Sprachen aufgezählt, die in der vorliegenden Tabelle (Abb. 2) abgebildet werden:

${ }^{15}$ In Österreich ist in den letzten Jahren die Debatte um eine Deutschpflicht in den Pausen und Schulhöfen, die oftmals mit einem Sprechverbot der Muttersprachen einhergeht, wieder aufgeflammt (siehe dazu auch Neuhold 2019; Tankır 2019). 


\section{F2: Welche Sprachen würdest du gerne lernen, die es an deiner Schule nicht gibt? (Sekundarstufe I)}

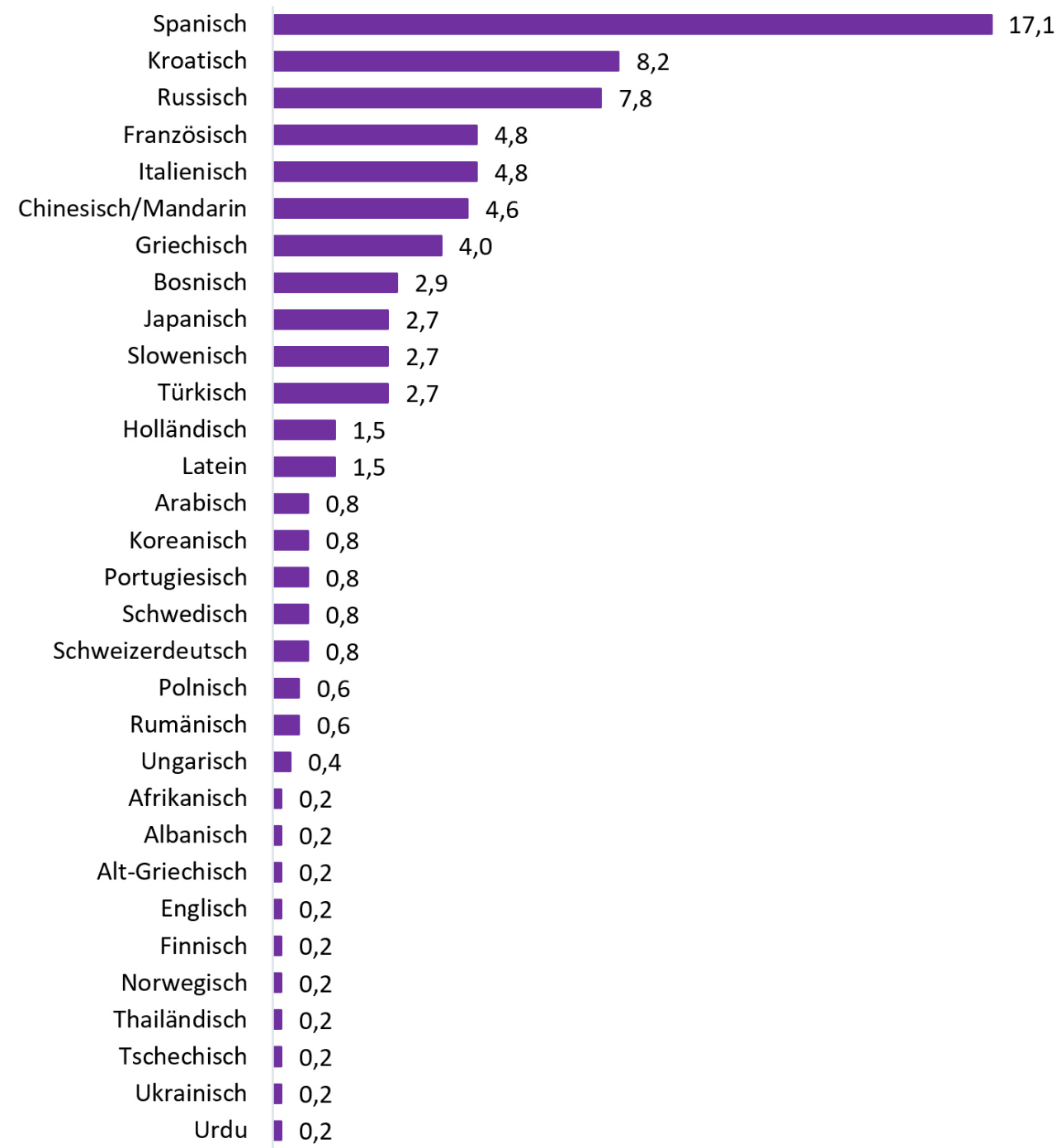

Abbildung 2: Sprachen, die es an den Schulen nicht gibt, die SchülerInnen der Sekundarstufe I jedoch gerne lernen würden, Angaben in Prozent (\%).

Die mit Abstand am häufigsten genannte Sprache in der Fragebogenerhebung ist „Spanisch" mit 17,1\%, das sind 81 SchülerInnen. Als Gründe für die Popularität dieser Sprache wurden einerseits ästhetische Bewertungen gemacht - Spanisch wird von den Befragten als „schön“ beschrieben, aber auch als „cool“, „toll“ und ,interessant“. Zudem ist Spanien ein beliebtes Urlaubsziel und daher haben einige SchülerInnen einen praktischen Grund als Motivation für das Erlernen dieser Sprache, nämlich die Verständigung 
im Urlaub, angeführt. Weitere Antworten der Befragten waren, dass ihnen die Kultur und das Land gefallen oder dass sie gerne in Spanien leben würden.

Nach Spanisch ist die Sprache, die von den meisten SchülerInnen genannt wurde, B/K/S (11,2\%), wobei hier „Kroatisch“ (8,2\%) und „Bosnisch“ (2,9\%) von den Befragten notiert wurden. In absoluten Zahlen sind das 53 SchülerInnen. Dieses Ergebnis ist bemerkenswert, da B/K/S zwar viele Mutter- und HerkunftssprecherInnen in Österreich hat, die Sprache selbst jedoch in der öffentlichen Wahrnehmung kaum präsent ist. SprecherInnen dieser Sprache werden, auch wenn sie perfekt integriert sind und Deutsch sprechen, häufig mit fremdenfeindlichen oder rassistischen Äußerungen konfrontiert. Eines der letzten Beispiele sind die Anfeindungen gegen die ursprünglich aus Bosnien stammende österreichische Politikerin Alma Zadić (vgl. Gindl 2020).

Während für Kroatisch sehr häufig ein praktischer Grund, nämlich der Urlaub in Kroatien, angegeben wurde, haben die meisten bei Bosnisch geschrieben, dass sie gerne ihre FreundInnen oder MitschülerInnen, die diese Sprache sprechen, verstehen und mit ihnen in dieser Sprache kommunizieren möchten. Eine Person hat zudem angegeben, dass sie Bosnisch lernen möchte, weil es ihre Muttersprache sei und eine Person möchte diese Sprache lernen, weil sie „bosnisch-kroatische Wurzeln” habe. Das bedeutet, dass bei B/K/S familiäre und persönliche Gründe eine besondere Rolle für die Motivation des Erlernens dieser Sprache darstellen. Die Antworten der Befragten weisen zudem darauf hin, dass bei B/K/S vor allem das Umfeld, der Freundeskreis sowie der Sportverein, für viele SchülerInnen ausschlaggebend dafür sind, diese Sprache lernen zu wollen. Weiters ist bei $\mathrm{B} / \mathrm{K} / \mathrm{S}$ ebenso die ästhetische Bewertung der Sprache für die Lernmotivation entscheidend - Kroatisch wird als „schöne“, Bosnisch dagegen vor allem als „coole“ Sprache charakterisiert.

An dritter Stelle der Sprachen, die in der Schule nicht angeboten werden, welche jedoch insgesamt 37 SchülerInnen gerne lernen würden, steht mit Russisch (7,8\%) eine weitere slawische Sprache. Als Grund für den Wunsch, diese Sprache zu lernen, haben die meisten Befragten angegeben, dass Russisch eine „coole“, „schöne“ oder „interessante"Sprache sei. Eine Person hat sogar geschrieben, Russisch sei „spannend“. Eine andere Person empfindet Russisch dagegen als eine Art ,Geheimsprache', da sie diese Sprache gerne lernen würde, um sich unterhalten zu können, ohne von den anderen verstanden zu werden. Auch Wirtschaft („Erfolg“, „Export“) sowie Sport („Eishockey“, „Volleyball“) wurden als Gründe für das Interesse an Russisch angeführt.

Weitere Sprachen, welche die SchülerInnen bei F2 genannt haben, sind die beiden romanischen Sprachen „Italienisch“ (4,8\%) und „Französisch“ (4,8\%), obwohl diese in den meisten Schulen im Lehrangebot bereits vorhanden bzw. Pflichtfächer sind. Während bei Französisch die meisten Befragten geschrieben haben, dass sie diese Sprache lernen 
wollen, weil sie „schön“ sei, war bei Italienisch der am häufigsten angeführte Grund ein praktischer - die Verständigung während des Urlaubs in Italien.

Zudem haben die Kinder bzw. Jugendlichen angegeben, dass sie gerne „Chinesisch“ (4,4\%) oder "Griechisch“ (4,0\%) lernen würden. „Chinesisch“ ist keine eigene Sprache, aber vulgo wird darunter die sinitische Sprache mit der größten Anzahl an SprecherInnen, nämlich Mandarin, impliziert. Ein einziger Schüler hat explizit „Mandarin“ und nicht „Chinesisch“ in seiner Antwort notiert. ${ }^{16}$ Chinesisch wird als „coole“, „schöne“ und „spannende“ Sprache empfunden. Neben Russisch ist Chinesisch die einzige Sprache, die mit dem Attribut „spannend“ charakterisiert wurde. Auch die „Schrift" sowie generell die „Herausforderung“ wurden als Beweggründe für das Erlernen dieser Sprache genannt. Griechisch wird ebenso als ,interessante“ und „coole "Sprache typisiert, von einer Person wurde Griechisch sogar als „elegante und schöne Sprache“ bezeichnet. Weitere Gründe für die Motivation, Griechisch zu lernen waren unter anderen der Urlaub, die Kultur sowie die Mythologie.

Eine weitere von einigen SchülerInnen in F2 aufgezählte Sprache ist "Slowenisch“ (2,7\%). Hier wurde als Hauptgrund die Nähe Kärntens zu Slowenien bzw. Slowenien als Nachbarland Österreichs hervorgehoben. Zwei Personen haben außerdem angegeben, dass sie viel Zeit in Slowenien verbringen und diese Sprache deshalb gerne lernen würden. Auch „Türkisch“ wollen einige Befragte lernen (2,7\%). Gründe dafür sind der Freundeskreis, der Urlaub in der Türkei sowie die Beschreibung der Sprache als „cool“ und ,interessant“.

Außerdem wurden auch „Holländisch“ (1,5\%) und „Latein“ (1,5\%) von den Jugendlichen angeführt. Während einige SchülerInnen „Holländisch“ lernen wollen, weil es ihre Muttersprache ist oder sie Bekannte in den Niederlanden haben, werden als Grund dafür, Latein lernen zu wollen, berufliche oder zukunftsorientierte Gründe (Arztberuf, Weiterbildung) genannt.

Auf die mit F2 in Verbindung stehende dritte Frage (F3), ob die SchülerInnen bereits eine der in F2 aufgezählten Sprachen außerhalb der Schule lernen, haben nur sehr wenige der Befragten positiv geantwortet. Demnach lernen lediglich 5,3\% der SchülerInnen, insgesamt 25 Personen, eine Sprache außerhalb der Schule, wobei aus den Antworten hervorgeht, dass die SchülerInnen keinen Unterschied zwischen dem bewussten (gesteuerten) language learning und der unbewussten (ungesteuerten) language acquisition gemacht haben. So lauteten die meisten Antworten der Befragten, dass sie eine der in F2 genannten Sprachen „zu Hause“, „mit den Eltern“ oder bei anderen Verwandten wie Großmutter, Großvater, Onkel oder Tante lernen. Dies fällt jedoch streng gesehen unter

\footnotetext{
${ }^{16}$ Die Antworten „Chinesisch“ und „Mandarin“ wurden in der Graphik (Abb. 2) als eine Sprache zusammengefasst.
} 
den ungesteuerten Spracherwerb (language acquisition) und nicht unter das bewusste gesteuerte Sprachlernen (language learning). Lediglich drei Personen haben auf die dritte Frage (F3) geantwortet, dass sie eine der in F2 aufgezählten Sprachen via „App“, „Handy“ oder „online“ lernen.

Die Antworten auf die einzige geschlossene Frage (F4) der vorliegenden Untersuchung sind in Bezug auf das Bundesland Kärnten aufschlussreich, da sie nicht nach den Wünschen der SchülerInnen fragt, sondern speziell nach dem Sprachangebot an Kärntner Schulen. Die vorliegende Tabelle stellt die Angaben der Befragten graphisch dar (Abb. 3):

\section{F4: Welcher Fremdsprachenunterricht sollte an Kärntner Schulen besucht werden können? (Sekundarstufe I)}

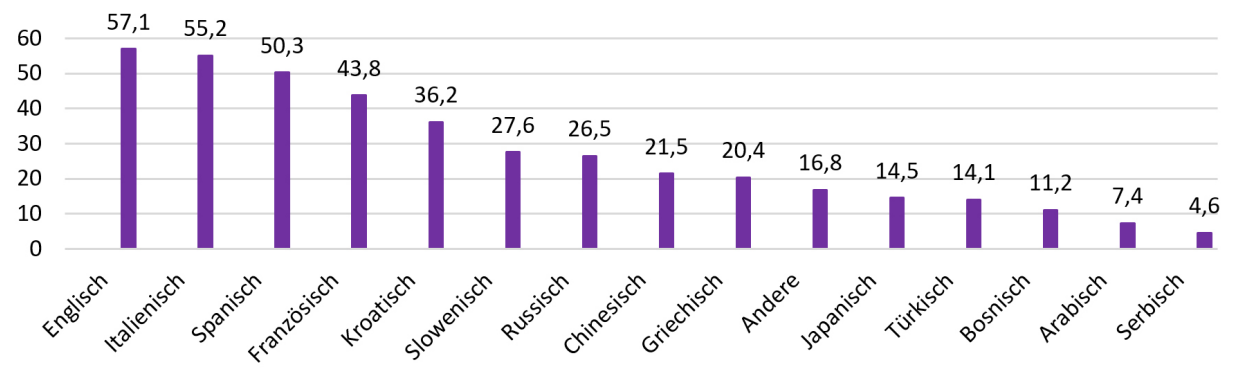

Abbildung 3: Fremdsprachenunterricht, der an Kärntner Schulen angeboten werden sollte (Sekundarstufe I), Angaben in Prozent (\%).

Wie die Graphik demonstriert, sind sich die Befragten einig, dass an Kärntner Schulen unbedingt Englisch $(57,1 \%)$ in den Schulen unterrichtet werden sollte. Diese Antwort spiegelt die Monopolstellung von Englisch an Österreichs Schulen sowie dessen globale Dominanz wider (vgl. Abschnitt 1.3).

An zweiter Stelle wurden Italienisch $(55,2 \%)$ und an dritter Stelle Bosnisch, Kroatisch und Serbisch, zusammengefasst B/K/S (52,0\%) genannt. Dieses Ergebnis weist einerseits sehr stark darauf hin, dass die SchülerInnen der Sekundarstufe I es wichtig finden, die Sprachen der Nachbarländer Kärntens zu lernen. Da Italienisch im Sprachangebot der meisten Schulen bereits existiert, ist es auch nicht verwunderlich, dass zuvor bei F3, die explizit nach Sprachen gefragt hat, welche noch nicht in der Schule unterrichtet werden, Italienisch mit 4,8\% nur von sehr wenigen Befragten als Sprache, die sie gerne lernen würden, genannt wurde.

Die Tatsache, dass sich B/K/S an dritter Stelle derjenigen Sprachen befindet, deren Unterricht nach Meinung der Befragten an Kärntner Schulen angeboten werden sollten, könnte darauf hinweisen, dass sich die SchülerInnen bewusst sind, dass viele Menschen in Österreich B/K/S sprechen und sie daher diese Sprache besser verstehen möchten. Die Antwort auf die fünfte Frage (F5) des Fragebogens bestärkt diese Vermutung, da 
„B/K/S“ die am häufigsten genannte Sprache (13,3\%) der MitschülerInnen ist, welche die Befragten gerne lernen würden.

Spanisch (50,3\%) wurde am vierthäufigsten bei der geschlossenen Frage (F4) angekreuzt; Französisch (43,8\%) liegt dagegen an fünfter Stelle. Spanisch ist, wie bereits die Antworten auf F2 gezeigt haben, sehr beliebt bei den SchülerInnen, und liegt bei der Fragebogenauswertung der Sekundarstufe I immer klar vor Französisch, dessen Stellenwert an österreichischen Schulen in den letzten Jahren deutlich abgenommen hat. Damit bestätigt die vorliegende Fragebogenerhebung Berichte aus den Medien, laut denen die Anzahl der SpanischlernerInnen an Österreichs Schulen deutlich zugenommen hat, während die Zahl der FranzösischlernerInnen kontinuierlich zurückgeht (vgl. Standard 2019). An sechster Stelle nach Häufigkeit liegt bei der vierten Frage Slowenisch (27,6\%). Slowenisch hat bei der Fragebogenerhebung der Sekundarstufe I im Gegensatz zu Italienisch oder B/K/S eher schlecht abgeschnitten, obwohl Slowenien auch ein Nachbarland Kärntens ist. Bei der fünften Frage, welche Sprache der MitschülerInnen die Befragten gerne lernen würden, hat Slowenisch ebenso nur 1,5\% erreicht.

Eine weitere Sprache, die nach Meinung von über einem Viertel der SchülerInnen der Sekundarstufe I an Kärntner Schulen unterrichtet werden sollte, ist Russisch (26,5\%). Russisch ist auch eine der Sprachen, die am häufigsten bei F5, der Sprache der MitschülerInnen, welche die SchülerInnen gerne lernen würden, genannt wurde. 11,2\% aller Befragten haben bei F5 angegeben, dass sie von den Sprachen der MitschülerInnen gerne Russisch lernen würden.

Weitere Sprachen, die an Kärntner Schulen laut Meinung der Befragten angeboten werden sollten, sind Chinesisch (21,5\%), Griechisch (20,4\%), Japanisch (14,5\%) und Türkisch (14,1\%). Hier wird deutlich, dass bei dieser Frage wahrscheinlich ebenso persönliche Interessen und praktische Gründe (z.B. Urlaub) die Antworten beeinflusst haben.

\subsection{Ergebnisse: Sekundarstufe II}

In der Sekundarstufe II haben 338 SchülerInnen an der Fragebogenerhebung teilgenommen, wobei hier festzuhalten ist, dass zwei der fünf untersuchten Schulen zweisprachig (Slowenisch und Deutsch) geführt werden und die Ergebnisse in Hinblick auf diese Besonderheit betrachtet werden müssen. Aufgrund dieser Tatsache ist es nicht verwunderlich, dass in der Sekundarstufe II 34,9\% der Befragten als Muttersprache „Deutsch“ und 59,2\% „Slowenisch“ angegeben haben. „Bosnisch“, „Kroatisch“, „Serbisch“ und „SerboKroatisch" haben zusammen 9,6\% der Befragten genannt und damit befindet sich B/K/S an dritter Stelle der Muttersprachen in den untersuchten Schulen der Sekundarstufe II. „Englisch“ haben 1,5\% als Muttersprache angegeben und alle übrigen genannten Mut- 
tersprachen wie „Albanisch“, „Arabisch“, „Bulgarisch“, „Chinesisch“, „Italienisch“, „Mazedonisch“, „Panjabi“ und „Ungarisch“ wurden von lediglich zwei oder weniger TeilnehmerInnen der Erhebung angegeben (=0,6\%). Über ein Achtel der Befragten (13\%) hat zudem zwei Muttersprachen angeführt. Dieses Ergebnis zeigt, dass auch die SchülerInnen der Sekundarstufe II mehrsprachig sind - neben Deutsch wurden 14 andere Sprachen ${ }^{17}$ als Muttersprachen angegeben.

Bei der ersten Frage (F1), welche Sprachen die SchülerInnen in der Schule lernen, liegt „Englisch“ (97,9\%) an erster und „Deutsch“ (94,1\%) an zweiter Stelle. Hier ist deutlich ein Unterschied zu den Ergebnissen der Sekundarstufe I zu erkennen, da Deutsch de facto fast genauso oft genannt wurde wie Englisch. Das könnte einerseits bedeuten, dass in der Sekundarstufe II mehr Bewusstsein unter den SchülerInnen herrscht, dass Deutsch auch zum Sprachunterricht zählt. Andererseits wurde Deutsch wahrscheinlich so häufig bei $\mathrm{F} 1$ aufgrund der Tatsache genannt, dass es für viele der Befragten tatsächlich eine Fremdsprache darstellt, da über 70\% der Jugendlichen eine andere Muttersprache als Deutsch oder neben Deutsch eine zweite Muttersprache angeführt haben.

An dritter Stelle der Sprachen, die in der Schule unterrichtet werden, befinden sich „Italienisch“ und an vierter Stelle „Slowenisch“. Außerdem lernen die SchülerInnen der Sekundarstufe II „Latein“ (12,7\%), „Russisch“ (3,8\%) sowie „B/K/S“ bzw. „Kroatisch“ (2,1\%). Interessant ist, dass hier zum ersten Mal bei den Antworten das Akronym „B/K/S“ notiert wurde, was wahrscheinlich damit zusammenhängt, dass der Sprachunterricht diese Bezeichnung trägt. Bei den Muttersprachen haben die SchülerInnen dagegen immer die jeweiligen Einzelsprachen angeführt. Auch „Spanisch“ (1,2\%) wurde bei den Fremdsprachen, die in der Schule bereits gelernt werden, aufgezählt.

Auf die zweite Frage, welche Sprachen die SchülerInnen gerne lernen würden, die es in der Schule nicht gibt, wurde wie auch in der Sekundarstufe I eine große Palette an Sprachen - insgesamt 30 Sprachen - aufgezählt. Besonders erwähnenswert ist, dass in der Sekundarstufe II als Antwort auf F2 die beiden Fantasiesprachen „Elbisch“ und „Valyrisch" genannt wurden, welche die SchülerInnen aus den Büchern und Filmen von Herr der Ringe und der Serie Game of Thrones kennen. Neben Sprachen wie „Afrikanisch“, „Italienisch“, „Norwegisch“, „Polnisch“, „Slowakisch“, „Tschechisch“, „Ungarisch“ oder „Urdu“, die jeweils lediglich von einer Person genannt wurden, kamen folgende Sprachen bei F2 vor (Abb. 4):

\footnotetext{
${ }^{17}$ Wenn wir „Bosnisch“, „Kroatisch“, „Serbisch“ und „Serbo-Kroatisch“ als eine Sprache zählen, sind es lediglich 11 verschiedene Muttersprachen, welche von den SchülerInnen genannt wurden.
} 


\section{F2: Welche Sprachen würdest du gerne lernen, die es an deiner Schule nicht gibt? (Sekundarstufe II)}

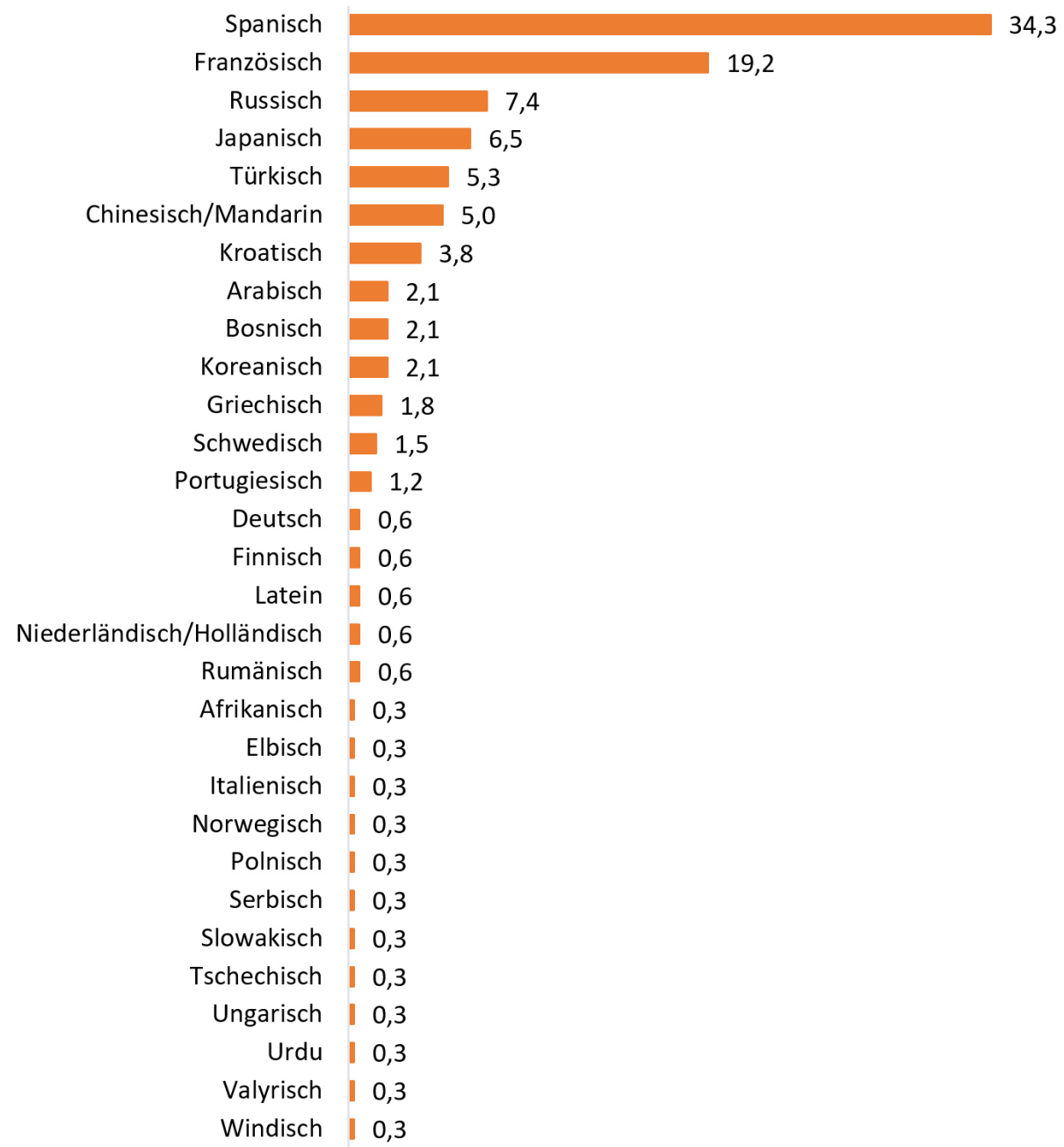

Abbildung 4: Sprachen, die es an den Schulen nicht gibt, die SchülerInnen der Sekundarstufe II jedoch gerne lernen würden, Angaben in Prozent (\%).

Hier ist noch einmal deutlicher als in der Sekundarstufe I die große Beliebtheit von Spanisch zu erkennen - mehr als ein Drittel der SchülerInnen (34,3\%), insgesamt 116 Befragte, hat angegeben, dass sie diese Sprache gerne in der Schule lernen würden. Der meistgenannte Grund für das Erlernen dieser Sprache ist ein ästhetischer - Spanisch wird auch von den SchülerInnen der Sekundarstufe II als „sehr schöne“ Sprache beschrieben. Zwei Personen haben in ihrer Antwort explizit auf die Prosodie der Sprache, die ihnen gefällt, verwiesen. Auffällig ist, dass in der Sekundarstufe II Spanisch auch emotional beschrieben wird. So geben fünf SchülerInnen an, dass sie diese Sprache „lieben“. Andere 
Gründe, welche die Befragten genannt haben, sind die Internationalität der Sprache, die hohe SprecherInnenzahl, die Kultur sowie Spanien als Urlaubsziel.

An zweiter Stelle derjenigen Sprachen, welche die SchülerInnen gerne lernen würden, steht Französisch (19,2\%), die insgesamt 65 Personen notiert haben. Auch hier wird als häufigster Grund die Schönheit der Sprache betont und einige Befragte haben geschrieben, dass sie diese Sprache oder Frankreich „lieben“. Außerdem würden die SchülerInnen gerne Französisch lernen, weil ihnen die Sprache „gefällt“ und sie „interessant“, „wichtig für die Berufswelt“" sowie die „Diplomatensprache“ sei.

Weit hinter Spanisch und Französisch liegt Russisch mit 25 Nennungen (7,4\%). Auch hier ist Grund für die Motivation, diese Sprache zu lernen, dass die Sprache „schön“, „cool“ und „interessant" sei. Außerdem wurde als Grund für das Erlernen dieser Sprache einige Male der Wunsch genannt, nach Russland zu reisen.

Fast gleichauf liegt das Interesse der SchülerInnen an Japanisch (6,5\%) und an B/K/S $(6,2 \%)$ mit jeweils knapp über 20 Nennungen. Als Gründe für Japanisch werden die Wichtigkeit, das Interesse an der Sprache sowie an der Schrift angeführt. Bei B/K/S werden praktische Gründe angeführt wie beispielsweise, dass es „gut für die Kommunikation sei“ oder weil „Freunde“ diese Sprache sprechen. Außerdem wird B/K/S als „,interessant" und „schön" beschrieben. Eine Person schrieb, dass sie diese Sprache zwar gerne lernen würde und an der Schule auch die Möglichkeit dazu hätte, jedoch fehle ihr aufgrund der hohen Auslastung an der Schule (Hausübungen, lernen) und außerschulischer Verpflichtungen die Zeit dazu. Die Fragebogenerhebung zeigt demnach auch, dass viele SchülerInnen keine neue bzw. zusätzliche Fremdsprache mehr in der Schule lernen wollen, weil sie dafür keine Zeit haben oder bereits, gesättigt' sind von den vielen unterschiedlichen Schulfächern. Daher haben einige SchülerInnen sowohl der Sekundarstufe I als auch der Sekundarstufe II als Antwort auf F2 angegeben, dass sie keine weitere Fremdsprache mehr lernen wollen.

Auch Türkisch (5,3\%) und Chinesisch (3,8\%) bzw. „Mandarin“ (1,2\%) wurden von den Jugendlichen in F2 genannt. Bei Chinesisch bzw. Mandarin wird hervorgehoben, dass diese Sprache eine „Weltsprache“ sei. Türkisch wollen die meisten Jugendlichen lernen, weil ihnen die Sprache "gefällt“ - auch hier wird in den Antworten explizit auf die Prosodie der Sprache (Melodie, „schöner Fluss“) hingewiesen. Ebenso haben die SchülerInnen als Antwort auf F2 „Arabisch“ (2,1\%) genannt, das die meisten als „interessant“ bezeichnen.

Auf die dritte Frage, ob die SchülerInnen bereits eine der in F2 genannten Sprache außerhalb der Schule lernen, haben 8,9\%, das sind insgesamt 28 Personen, positiv geantwortet. Am häufigsten wird „Spanisch“ (2,7\%), nämlich von neun Befragten, außerhalb der Schule gelernt. Auch „Französisch“ (0,9\%), „Japanisch“ (0,6\%), „Koreanisch“ 
$(0,6 \%)$ und „Bosnisch“ (0,6\%) wurden von einigen Jugendlichen genannt. Im Gegensatz zu den SchülerInnen aus der Sekundarstufe I, welche hier auch den Spracherwerb (language acquisition) zuhause als Beispiele gebracht haben, nannten viele SchülerInnen der Sekundarstufe II Sprachlern-Apps für das Handy (v.a. Duolingo, Babble und Memrise), mithilfe derer sie die in F2 genannten Sprachen lernen. Auch das Lernen von Sprachen mithilfe von Fernsehen und Serien wurde einige Male angeführt. Nur zwei Befragte haben angegeben, dass sie Französisch bzw. Spanisch zusätzlich privat lernen. Die Antworten der Sekundarstufe II in Bezug auf das Sprachangebot speziell an Kärntner Schulen unterscheiden sich von jenen der Sekundarstufe I. Die nachfolgende Graphik (Abb. 5) illustriert die Antworten der Sekundarstufe II auf die vierte Frage (F4) der vorliegenden Erhebung:

\section{F4: Welcher Fremdsprachenunterricht sollte an Kärntner Schulen besucht werden können? (Sekundarstufe II)}

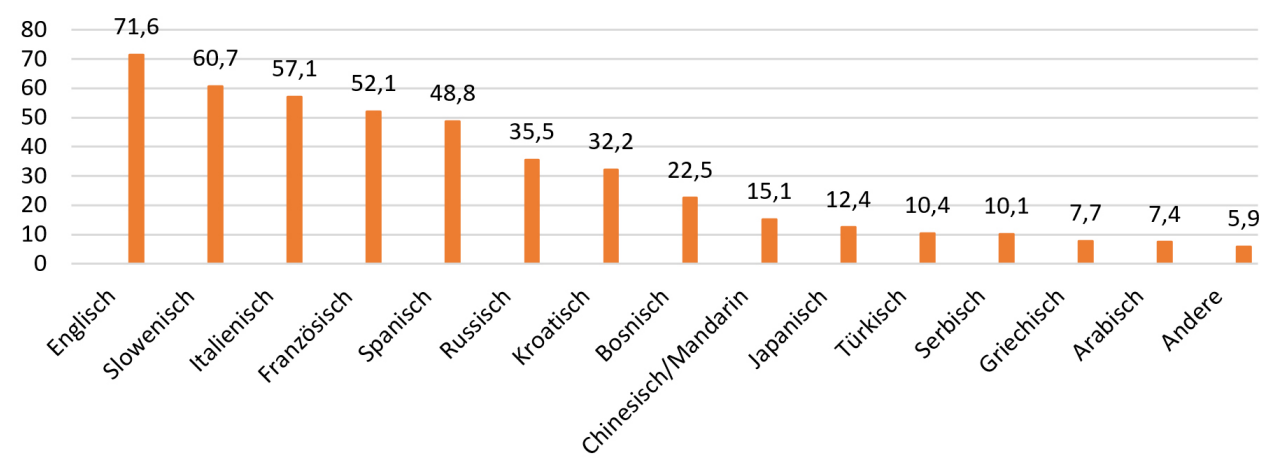

Abbildung 5: Fremdsprachenunterricht, der an Kärntner Schulen angeboten werden sollte (Sekundarstufe II), Angaben in Prozent (\%).

Wie in der Sekundarstufe I gab die Mehrheit der Befragten zwar an, dass Englisch $(71,6 \%)$ die Sprache sei, die an Kärntner Schule angeboten werden sollte. An zweiter Stelle wurden jedoch B/K/S (64,8\%) und an dritter Stelle Slowenisch (60,7\%) genannt. Diese beiden slawischen Sprachen liegen in der Sekundarstufe II daher klar vor den romanischen Sprachen Italienisch $(57,1 \%)$ und Französisch $(52,1 \%)$. Das könnte damit zusammenhängen, dass viele TeilnehmerInnen der Erhebung in der Sekundarstufe II Slowenisch als Muttersprache haben und in eine zweisprachige Schule gehen. Das Slowenische könnte aus diesem Grund generell bei diesen SchülerInnen präsenter sein und als wichtiger empfunden werden als bei ihren KollegInnen in der Sekundarstufe I.

Auch die Antworten auf die fünfte Frage (F5), welche Sprache der MitschülerInnen die Befragten gerne lernen würden, weisen darauf hin, dass einige der untersuchten Schulen zweisprachig sind, da 22,8\% als Sprache ihrer MitschülerInnen gerne „Deutsch“ ler- 
nen würden und 8,3\% „Slowenisch“. Der weitaus größte Teil, nämlich 22,2\% würde jedoch von den Sprachen ihrer MitschülerInnen gerne B/K/S lernen. Damit liegt B/K/S („Bosnisch“, „Kroatisch“ oder „Serbisch“) wie auch in der Sekundarstufe I bei F5 an der Spitze.

In Bezug auf F4 fällt außerdem auf, dass Spanisch zwar sehr weit vorne liegt, wenn die Frage nach den persönlichen Wünschen der SchülerInnen gestellt wird wie in F2, aber, dass Spanisch (48,8\%) hinter B/K/S, Slowenisch, Italienisch und sogar Französisch liegt, wenn es speziell um das Sprachangebot an Kärntner Schulen geht.

Russisch hat auf die Frage, welche Fremdsprachen an Kärntner Schulen angeboten werden sollen, 35,5\% erreicht und liegt damit deutlich vor anderen Sprachen wie Chinesisch (15,1\%), Japanisch (12,3\%) oder Türkisch (10,4\%). Dennoch zeigen die Ergebnisse der Fragebogenerhebung in der Sekundarstufe II deutlich, dass, wenn es um das Sprachangebot an Kärntner Schulen geht, die Präferenz klar den Sprachen des Alpen-AdriaRaumes gegeben wird.

\section{Zusammenfassung und Ausblick}

Die vorliegende großteils quantitative Erhebung, die mittels eines Fragebogens durchgeführt wurde, gibt einen ersten Eindruck über das Sprachangebot an Kärntner Schulen und die sprachlichen Wünsche und Vorstellungen der SchülerInnen. Die Untersuchung stellt lediglich eine Pilotstudie dar, da die Fragebogenerhebung in nur fünf von insgesamt über 50 allgemein- und berufsbildenden höheren Schulen durchgeführt wurde. Dennoch spiegeln die Ergebnisse dieser Pilotstudie allgemeine Beobachtungen wider und sind daher aus mehreren Gründen interessant: Erstens zeigen sie deutlich die Monopolstellung des Englischen, was nicht weiter verwunderlich ist, da Englisch nicht nur die wichtigste Fremdsprache in Österreichs Schulen ist, sondern auch die wichtigste Fremdsprache auf internationaler Ebene. Zweitens bestätigt die Untersuchung deutlich den Trend, dass zurzeit Spanisch die favorisierte Fremdsprache vieler SchülerInnen ist und der Stellenwert von Französisch deutlich abgenommen hat. Drittens, und das ist wahrscheinlich das wichtigste Ergebnis dieser Studie, demonstriert sie das Interesse der SchülerInnen an einem Fremdsprachenunterricht in slawischen Sprachen, allen voran an Bosnisch/Kroatisch/Serbisch. Das Resultat der Erhebung weist darauf hin, dass $\mathrm{B} / \mathrm{K} / \mathrm{S}$ sowohl in der Sekundarstufe I als auch in der Sekundarstufe II diejenige Sprache ist, welche neben Italienisch und Slowenisch an Kärntner Schulen angeboten werden sollte. Zudem ist B/K/S die am häufigsten genannte Sprache, wenn es um die Sprache der MitschülerInnen geht, welche die SchülerInnen gerne lernen würden. 
Angesichts dieser Resultate, die als erste Einblicke in das Sprachangebot an Kärntner Schulen gesehen werden können, müsste die vorliegende Studie vor allem qualitativ ausgeweitet werden, um differenziertere Aussagen über die jeweiligen Gründe und tatsächlichen Motivationen der SchülerInnen in Bezug auf das Sprachenlernen in der Schule treffen zu können. Wichtig wäre vor allem eine Ausweitung der Untersuchung auf die Schulen, die bei der Interessenserhebung unter DirektorInnen angegeben haben, dass kein Interesse an Sprachen wie B/K/S oder Russisch besteht, da diese Angaben deutlich im Widerspruch zu den Ergebnissen der vorliegenden Pilotstudie stehen. Gerade hier wäre es einerseits notwendig herauszufinden, ob und warum kein Interesse an diesen Sprachen besteht. Anderseits wäre es aufschlussreich auch die Betroffenen, nämlich die SchülerInnen, nach ihren sprachlichen Präferenzen zu befragen, um so die Ergebnisse der vorliegenden Studie zu stärken.

\section{Literatur}

Atteslander, Peter. 2010. Methoden empirischer Sozialforschung. 13. neu bearb. und erw. Aufl. Berlin: Erich Schmidt.

Aygün-Sagdic, Gülden, Oana Bajenaru und Claus Melter. 2015. „Gedanken zum Verhältnis von Rassismus, nationalsprachlicher Diskriminierung und Neolinguizismus." In Sprache und Bildung in Migrationsgesellschaften. Machtkritische Perspektiven auf ein prekarisiertes Verhältnis (= Reibe: Kultur und soziale Praxis), [ebook], hrsg. von Nadja Thoma und Magdalena Knappik, 109-129. Bielefeld: transcript.

Beer, Romana. 2019. „Mehrsprachigkeit zu fördern, ist ein gesellschaftliches Muss.“ In stimme. Zeitschrift für Initiative Minderbeiten 113: 14-15.

BMBWF. 2020a. = Bundesministerium für Bildung, Wissenschaft und Forschung. 2020a. „Sprachliche Bildung."Wien. Abgerufen am 07.Juni 2020. https://www.bmbwf.gv.at/Themen/schule/ schulpraxis/ba/sprabi.html.

BMBWF. 2020b. = Bundesministerium für Bildung, Wissenschaft und Forschung. 2020b. „Fremdsprachenlernen."Wien. Abgerufen am 07.Juni 2020. https://www.bmbwf.gv.at/Themen/schule/ schulpraxis/ba/sprabi/fms.html.

Bunčić, Daniel. 2008. „Die (Re-)Nationalisierung der serbokroatischen Standards.“ In Deutsche Beiträge zum 14. Internationalen Slawistenkongress Ohrid 2008, hrsg. von Sebastian Kempgen, 89-102. Wiesbaden: Harrassowitz.

Bundeskanzleramt. 2015. 4. „Bericht der Republik Österreich gemäß Artikel 15 Abs. 1 der Europäischen Charta der Regional- oder Minderheitensprachen." Wien: Bundeskanzleramt, Bundespressedienst. Abgerufen am 07. Juni 2020. https://rm.coe.int/168070c2f3.

Busch, Brigitta. 2010. „Slowenisch in Kärnten - Sprache jenseits ethnischer Kategorien“. In Grenzverkehr/ungen. Mehrsprachigkeit, Transkulturalität und Bildung im Alpen-Adria-Raum, hrsg. von Werner Wintersteiner, Georg Gombos und Daniela Gronold, 174-188. Klagenfurt: Wieser. 
Busch, Brigitta. 2016. „Wenn der Krieg ins Klassenzimmer eindringt - Ein Blick zurück aus aktuellem Anlass." In In die Welt hinaus. Festschrift für Renate Feistauer zum 65. Geburtstag, hrsg. von Hannes Schweiger, Vera Ahamer, Clemens Tonsern, Tina Welke und Nadja Zuzok, 165175. Wien: Praesens.

Carré-Carlinger, Catherine, Dagmar Gilly, Michaela Haller, Martina Huber-Kriegler, Eva Vetter und Alexandra Wojnesitz. 2014. Basiskompetenzen Sprachliche Bildung für alle Lehrenden. Graz: ÖSZ. Abgerufen am 07. Juni 2020. http://oesz.at/OESZNEU/UPLOAD/Basiskom petenzen_sprachliche_Bildung_FINAL.pdf.

Corazza, Jennifer. 2016. Der Begriff Muttersprache - Eine kritische Analyse anhand literarischer Sprachbiografien, Master thesis. Wien: Universität Wien.

Council of Europe. 2020. "Reservations and Declarations for Treaty No. 148 - European Charter for Regional or Minority Languages.” Austria. Abgerufen am 07. Juni 2020. https://tinyurl. com/yda9ahjz.

De Cillia, Rudolf und Ruth Wodak. 2006. Ist Österreich ein „deutsches"Land? Sprachenpolitik und Identität in der Zweiten Republik. Innsbruck [u.a.]: Studienverlag.

De Cillia, Rudolf. 2020. „Sprache/n und Identität/en. In Österreichische Identitäten im Wandel. Empirische Untersuchungen zu ihrer diskursiven Konstruktion 1995-2015, [ebook], hrsg. von Rudolf De Cillia, Ruth Wodak, Markus Rheindorf und Sabine Lehner, 81-114. Wiesbaden: Springer.

Diekmann, Andreas. 2014. Empirische Sozialforschung. Grundlagen, Methoden, Anwendungen. Reinbek bei Hamburg: Rowohlt.

Doleschal, Ursula. 2012. „Österreich (Republik Österreich).“ In Europäische Charta der Regionaloder Minderheitensprachen. Ein Handbuch zur Sprachpolitik des Europarats, hrsg. von Franz Lebsanft, 191-209. Berlin: de Gruyter.

Europäische Kommission, ed. 2012. Die europäischen Bürger und ihre Sprachen. Spezial Eurobarometer 386. Abgerufen am 07. Juni 2020. https://ec.europa.eu/commfrontoffice/publicopinion/ archives/ebs/ebs_386_de.pdf.

Garnitschnig, Ines. 2019. „Der muttersprachliche Unterricht in Österreich. Statistische Auswertung für das Schuljahr 2017/18." In Informationsblätter zum Thema Migration und Schule, Nr. 5, 1-42, hrsg. von Bundesministerium für Bildung, Wissenschaft und Forschung. Abgerufen am 07. Juni 2020. http://www.schule-mehrsprachig.at/fileadmin/schule_mehrsprachig/ redaktion/hintergrundinfo/info5_190418.pdf.

Gindl, Barbara. 2020. „Ministerkandidatin Zadić unter Beschuss“. Die Presse [online], 7. Januar 2020. Abgerufen am 07. Juni 2020. https://www.diepresse.com/5747731/ministerkandidatinzadic-unter-beschuss.

Grandovska, Baiba und Elisabeth Rohner-Thielen. 2017. „60\% der Schüler im Sekundarbereich I erlernten 2015 mehr als eine Fremdsprache. Französisch an zweiter Stelle nach Englisch." Eurostat Pressemitteilung 33/2017. Abgerufen am 07. Juni 2020. https://ec.europa.eu/eurostat/ documents/2995521/7879488/3-23022017-AP-DE.pdf.

Krumm, Hans-Jürgen. 2011. „Die deutsche Sprache und die Mehrsprachigkeit in Europa - ein sprachenpolitischer Blick auf Deutsch als europäische Sprache“. In Europasprachen, hrsg. von Peter Cichon und Michael Mitterauer, 99-112. Wien: Böhlau. 
Krumm, Hans-Jürgen und Hans H. Reich. 2011. „Curriculum Mehrsprachigkeit.“ Abgerufen am 04. Juni 2020. http://oesz.at/download/cm/CurriculumMehrsprachigkeit2011.pdf.

Neuhold, Petra. 2019. „,Muttersprachlicher “ Unterricht. Eine Erinnerung.“ In stimme. Zeitschrift der Initiative Minderheiten 113: 10-11.

OeAD. 2018. „OeAD-Mobilitätsradar Bildung 2017“. Abgerufen am 07. Juni 2020. https://oead. at/fileadmin/Dokumente/oead.at/KIM/OeAD/20180621_OeAD_Mobilitaetsradar_Bildu ng_2017_alle_Bereiche.pdf.

ÖSZ, BMUKK und BMWF, hrsg. 2009. Sprach- und Sprachunterrichtspolitik in Österreich. Länderprofil. Language and Language Education Policy in Austria. Country Report. Graz/ Wien: ÖSZ. Abgerufen am 07.Juni 2020. https://rm.coe.int/sprach-und-sprachunterrichtspo litik-in-osterreich-landerprofil/16807b3b9c.

Pederin, Ivan. 1995. „Austrijska jezična politika u Hrvatskoj i briga za standardizaciju hrvatskog jezika u Dalmaciji.“ In Jezik: časopis za kulturu hrvatskoga književnog jezika 43 (3): 91-103.

Purkarthofer, Judith und Brigitta Busch. 2013. „Einleitung und Zielsetzung.“ In Schulsprachen Sprachen in und um und durch die Schule (= Schulheft 151/2013), hrsg. von Judith Purkarthofer und Brigitta Busch, 5-8. Innsbruck: Studienverlag.

Riehl, Claudia M. 2013. „Mehrsprachigkeit und Sprachkontakt.“ In Sprachwissenschaft: Grammatik - Interaktion - Kognition, hrsg. von Peter Auer, 377-404. Stuttgart/Weimar: J.B.Metzler.

Rienzner, Martina. 2013. „Platz machen“ und Schule (mit)gestalten. Muttersprachlicher Unterricht in Somali." In Schulsprachen - Sprachen in und um und durch die Schule (= Schulheft 151/2013), hrsg. von Judith Purkarthofer und Brigitta Busch, 28-42. Innsbruck: Studienverlag.

RIS. 2020a = Rechtsinformationssystem des Bundes. „Bundesrecht konsolidiert: Gesamte Rechtsvorschrift für Lehrpläne - allgemeinbildende höhere Schulen, Fassung vom 07.06.2020“ Abgerufen am 07. Juni 2020. https://www.ris.bka.gv.at/GeltendeFassung.wxe?Abfrage=Bundes normen\&Gesetzesnummer $=10008568$.

RIS. 2020b = Rechtsinformationssystem des Bundes. „Bundesrecht konsolidiert: Gesamte Rechtsvorschrift für Lehrpläne - Neue Mittelschulen, Fassung vom 07.06.2020“' Abgerufen am 07. Juni 2020. https://www.ris.bka.gv.at/GeltendeFassung.wxe?Abfrage=Bundesnormen\&Gese tzesnummer $=20007850$.

Schreger, Christian. 2013. „Die gespaltene Zunge.“ In Schulsprachen - Sprachen in und um und durch die Schule (= Schulheft 151/2013), hrsg. von Judith Purkarthofer und Brigitta Busch, 9-15. Innsbruck: Studienverlag.

Standard. 2019. „Fremdsprachenunterricht: Spanisch auf Vormarsch, Französisch baut ab.“ Der Standard [online], 18. Januar 2019. Abgerufen am 16. Februar 2020. https://www.derstandard. at/story/2000096512644/fremdsprachenunterricht-franzoesisch-baut-ab-spanisch-legt-zu.

Statistik Austria. 2019. „Fremdsprachenunterricht der Schülerinnen und Schüler im Schuljahr 2017/18. Schulstatistik." Abgerufen am 07.Juni 2020. https://www.statistik.at/wcm/idc/idcplg? IdcService=GET_NATIVE_FILE\&RevisionSelectionMethod $=$ LatestReleased $\& \mathrm{dDocName}=$ 064757.

Tankır, Mehmet F. 2019. „Fördert die Deutschpflicht im Pausenhof die Bildungssprache Deutsch?“ In stimme. Zeitschrift der Initiative Minderheiten 113: 12-13. 
Wolf, Michaela. 2012. Die vielsprachige Seele Kakaniens. Übersetzen und Dolmetschen in der Habsburgermonarchie 1848 bis 1918. Abgerufen am 10. Juli 2020. Wien: Böhlau. https://services.ebook.fwf.ac.at/api/object/o:18/diss/Content/get. 